\section{CARACTERIZACIÓN DE FRUTOS DE CIRUELA MEXICANA (Spondias purpurea L.) DEL SUR DE MÉXICO}

\section{FRUIT CHARACTERIZATION OF MEXICAN PLUM (Spondias purpurea) FROM SOUTHERN MÉXICO}

\author{
Irán Alia-Tejacal ${ }^{1 \star}$, Yanik I. Astudillo-Maldonado ${ }^{1}$, \\ Carlos A. Núñez-Colín ${ }^{2}$, Luis A. Valdez-Aguilar, \\ Silvia Bautista-Baños ${ }^{4}$, Enrique García-Vázquez ${ }^{5}$, \\ Rafael Ariza-Flores ${ }^{6}$ y Fernando Rivera-Cabrera ${ }^{7}$
}

\begin{abstract}
${ }^{1}$ Postgrado en Ciencias Agropecuarias y Desarollo Rural, Facultad de Ciencias Agropecuarias, Universidad Autónoma del Estado de Morelos. Av. Universidad 1001, Col. Chamilpa, 62209, Cuernavaca, Morelos. ${ }^{2}$ Campo Experimental Bajío, Instituto Nacional de Investigaciones Agrícolas, Forestales y Pecuarias. km. 6.5 Carr. Celaya-San Miguel Allende. 38110, Celaya, Gto. ${ }^{3}$ Departamento de Horticultura, Universidad Autónoma Agraria Antonio Navaro. Blvd. Antonio Narro s/n. 25315, Buenavista, Saltillo, Coahuila. ${ }^{4}$ Centro de Desarrollo de Productos Bióticos, Instituto Politécnico Nacional. km.8.5 Carr. Yautepec-Jojutla. 62730, Yautepec, Morelos. ${ }^{5}$ Campo Experimental Las Huastecas, INIFAP. Col. Estacion Cuauhtemoc, km. 55 Carr. Tampico-Mante. 89610, Altamira Tamaulipas $\mathrm{y}^{6} \mathrm{Campo}$ Experimental Chilpancigo, INIFAP. A. Rufo Figueroa s/n, Col. Burócratas. 39039, Chilpancingo, Guerrero. ${ }^{7}$ Universidad Autónoma Metropolitana. Av. San Rafael Atlixco 186, Col. Vicentina. 09340, Iztapalapa, México, D.F.
\end{abstract}

*Autor para correspondencia (ijac96@yahoo.com.mx)

\section{RESUMEN}

Sesenta y siete colectas de ciruela mexicana (Spondias purpurea L.) de los Estados de Guerrero, Morelos y Chiapas fueron evaluadas en sus características de fruto en masa, dimensiones, componentes del color $\left(\mathbf{L}^{*}, \mathrm{C}^{*} \mathrm{y} \mathbf{H}^{*}\right.$ ), sólidos solubles totales (SST), acidez titulable (AT) y la proporción SST/AT. La masa del fruto, el ángulo matiz $\left(\mathrm{H}^{*}\right)$ y la proporción SST/AT tuvieron los mayores coeficiente de variación (CV: 50-60 \%) y contribuyeron a la formación de siete grupos. La masa de los frutos varió de 4.0 a $43.2 \mathrm{~g}$; el color desde rojo $\left(\mathrm{H}^{*}=15\right)$ hasta con tendencia al verde $\left(\mathrm{H}^{*}=105\right)$, mientras que la SST/AT varió entre 3.0 y 63.2; está última debido a la variación en SST (entre 3.2 y 17.3 ${ }^{\circ} \mathrm{Brix}$ ) y en AT (entre 0.2 y $2.0 \%$ ). Estos resultados evidencian una alta variabilidad entre las colectas estudiadas y alguna con potencial para su utilización hortícola.

Palabras clave: Spondias purpurea, masa, sólidos solubles totales, ángulo matiz, acidez titulable.

\section{SUMMARY}

Sixty seven Mexican plum (Spondias purpurea L.) accessions native to states of Guerrero, Morelos and Chiapas were evaluated regarding fruit mass, dimensions, color components $\left(\mathrm{L}^{*}, \mathrm{C}^{\star} \mathrm{y} \mathrm{H}^{*}\right)$, total soluble solids (TSS), titratable acidity (TA) and the TSS/TA ratio. The highest coefficient of variation (50-60 \%) was observed in fruit mass, hue angle $\left(\mathrm{H}^{*}\right)$ and the TSS/TA ratio, which contributed to the formation of seven groups. Fruit mass varied from 4.0 to $43.2 \mathrm{~g}$, fruit color from red $\left(\mathrm{H}^{\star}=\right.$
$15)$, to green $\left(H^{*}=105\right)$, whereas the TSS/TA ratio ranged from 3.0 to 63.2, due to the variation in TSS (from 3.2 to $17.3^{\circ} \mathrm{Brix}$ ) and TA (from 0.2 to $2.0 \%$ ). These results show the high variabilityamong accessions, some of them having potential for horticultural use.

Index words: Spondias purpurea, mass, total soluble solids totals, hue angle, titratable acidity.

\section{INTRODUCCIÓN}

La familia Anacardiaceae comprende más de 70 géneros con 600 especies. La familia se subdivide en 5 tribus: Anacardiae, Spondiadeae, Rhoeae, Semecarpeae y Donieeae. Algunos integrantes de Anacardiae y Rhoeae como el marañón (Anacardium occidentale L.), mango (Mangifera indica), sumac (Rhus sp.) y pistache (Pistacia vera L.) son alimentos vegetales importantes (Macía y Barfod, 2000). La tribu Spondiadeae incluye al género Spondias, el cual incluye 17 especies, siete de las cuales son neo tropicales y diez del trópico asiático (Ruenes et al., 2010). Las especies de ciruela mexicana (Spondias purpurea L.), jobo (S. mombin L.) y jocote (S. radkoferi J. D. Smith), son consideradas nativas de México y forman parte de las selvas bajas caducifolias y subcaducifolias; se distribuyen naturalmente a lo largo de la costa occidental y la zona sureste del país (Avitia et al., 2000). S. purpurea y S. mombin son las más conocidas y consumidas desde la época prehispánica (Ramírez et al., 2008). Aunque estas especies se han introducido al cultivo con fines comerciales, la información de los diferentes genotipos presentes es escasa, debido principalmente a que su cultivo está basado en la agricultura informal como huertos de traspatio, cercas vivas y granjas pequeñas, y a que crece de manera silvestre en zonas de difícil acceso.

Los árboles de Spondias purpurea son utilizados como cercos vivos, sus hojas son muy apetecibles por el ganado, y su fruto se consume en estado inmaduro o maduro (Ramírez et al., 2008). En algunas regiones como Chiapas es cultivado en forma comercial con un incipiente manejo agronómico que involucra fertilización y control de plagas. Los frutos de la ciruela mexicana son drupas oblongas, redondas u ovoides; de tamaños y masas diversas que fluctúan entre 2 y $5 \mathrm{~cm}$ y entre 4 y $33 \mathrm{~g}$ respectivamente, con epicarpio liso a semiliso, delgado y de coloración roja, amarilla, café rojiza, anaranjada o morada al madurar, con endocarpio grueso y fibroso y mesocarpio de sabor y aroma agradables (Avitia et al. 2000). El fruto maduro de la ciruela mexicana proporciona alta densidad calórica, vitamina $\mathrm{C}$ y moderada proporción de minerales como potasio y calcio (Koziol y Macía, 1998), así como compuestos antioxidantes como fenoles (Beserra et al., 2011) y carotenoides.

La ciruela mexicana es considerada como un frutal con alto potencial por su bajo costo de producción, porque crece en forma espontánea y se adapta a suelos pobres y delgados donde no se establecen otros cultivos; además 
posee resistencia a sequía mediante defoliación (Avitia et al., 2000). Según Ramírez et al. (2008), puede ser considerada como una especie frutal estratégica para la agricultura en regiones de México donde en la primavera (periodo seco del año) no hay otros frutales en producción, cuando alcanza precios relativamente altos.

No obstante la importancia de este recurso genético, la investigación en México en torno a su diversidad genética y manejo agronómico es escasa. Los aspectos estudiados se han enfocado a caracterización poscosecha, como el uso de películas comestibles (Bautista-Baños et al., 2003, Bautista et al., 2006), refrigeración (Pérez, 2004; Nava et al., 2010), y respuesta a la aplicación de retardadores de la maduración (Osuna et al., 2011). También se han caracterizado los sistemas de producción, etnobotánica y ecotipos sobresalientes en Jalisco, Colima y Nayarit (Ramírez et al., 2008), Veracruz (Nava-Kuri y Uscanga, 1979), Tabasco (VargasSimón et al., 2011) y Yucatán (Ruenes-Morales et al. 2010).

En los Estados de Guerrero y Morelos, hay áreas donde la ciruela mexicana crece en forma silvestre, fomentada o de manera comercial, pero poco se han documentado las características físicas, químicas y morfológicas de estos materiales, por lo que este es un trabajo inicial de caracterización de los frutos de ciruela mexicana en esta región.

\section{MATERIALES Y MÉTODOS}

Durante abril y mayo de 2010 se realizaron recorridos en 10 municipios del sur del Estado de Morelos y dos municipios del norte del Estado de Guerrero (Cuadro 1), donde se colectaron 30 frutos de cada árbol en madurez fisiológica o madurez de consumo, que fueron trasladados al laboratorio para su evaluación. La localización de cada colecta se determinó con un GPS Garmin Etrex ${ }^{\circledR}$ y se reporta en el Cuadro 1. Adicionalmente se obtuvieron ocho materiales de Chiapas adquiridos en la Central de Abastos del Distrito Federal. En total se evaluaron 67 colectas, 8 de Chiapas, 13 de Guerrero y 46 de Morelos.

Los frutos fueron lavados con agua destilada y secados a temperatura ambiente; luego se seleccionaron 10 frutos que no tuvieran daños físicos o por patógenos, para medir sus características: masa con una balanza digital (OHAUS $\AA$, USA) con una sensibilidad de $0.01 \mathrm{~g}$; diámetro polar y longitudinal, con un vernier (Mitutoyo ${ }^{\circledR}$, Japan) con una sensibilidad de $0.01 \mathrm{~mm}$; color de la epidermis con un espectrofotómetro (X-rite mod. 3690®, USA) en dos partes opuestas de la sección ecuatorial de cada fruto y se realizaron dos mediciones para obtener los datos de Luminosidad $\left(\mathrm{L}^{*}\right)$, cromaticidad $\left(C^{*}=\sqrt{\left(a^{\star}\right)^{2}+\left(b^{*}\right)^{2}}\right)$ y matiz $\left(H^{*}=\tan ^{-1} b^{*} / a^{*}\right)$ (McGuire, 1992); sólidos solubles totales
(SST) en una gota de jugo de la pulpa de cada fruto, con un refractómetro Atago PAL-1 ${ }^{\circledR}$ (Japan) en unidades ${ }^{\circ}$ Brix; y acidez titulable en muestras de $10 \mathrm{~g}$ de pulpa que se molieron con agua destilada, cuyo macerado se filtró con manta de cielo, y del filtrado se tomaron alícuotas de $5 \mathrm{~mL}$, a los que se agregaron dos gotas de fenolftaleína (1\%) y luego se tituló con hidróxido de sodio $0.1 \mathrm{~N}$. Con los valores de las dimensiones se obtuvo un índice de forma, resultante de dividir diámetro polar entre diámetro longitudinal del fruto (Gaona-García et al., 2008); con los atributos químicos se obtuvo un índice de sabor dividiendo los SST entre la AT (Ladaniya, 2008).

Con los datos obtenidos se hicieron análisis de estadística descriptiva de las poblaciones evaluadas, mediante el procedimiento UNIVARIATE de SAS (SAS Institute, 1998). Luego los datos se sometieron a un análisis multivariado de agrupamiento, con el Sistema de Taxonomía Numérica (NTSYSpc 2.1); para el agrupamiento se utilizó el procedimiento de agrupamiento secuencial, aglomerativo, jerárquico y anidado (SAHN), con el que se construyó un dendograma, con el método de ligamiento promedio (UPGMA), para generar el dendograma se utilizó la distancia taxonómica promedio.

\section{RESULTADOS Y DISCUSIÓN}

La masa del fruto en la población estudiada fluctuó entre 4.0 y 43.2 g (Cuadro 2). El valor mayor lo presentó la colecta $\mathrm{CH} 3$ proveniente de Chiapas, denominada 'Jocote grande'; los materiales con masa menor fueron GRO 6 y MOR64, de color rojo dulce y acida respectivamente. NavaKuri y Uscanga (1979), reportaron valores entre 8.7 y 37 g en 12 materiales de ciruela mexicana en Veracruz, en tanto que Ramírez et al. (2008) indicaron valores entre 6.3 y 35.8 $\mathrm{g}$ en frutos colectados en Colima, Jalisco y Nayarit. Vargas-Simón et al. (2011) caracterizaron cuatro materiales en Tabasco con masa promedio entre 15.5 y 24.9 g. Según Ramírez et al. (2008), los ecotipos cultivados tienen frutos de tamaño mayor que los silvestres.

El diámetro polar y logitudinal y el cociente entre ellos mostraron bajos coeficientes de variación (entre 6.0 y 22.0 $\%$; Cuadro 2). Ramírez et al. (2008), reportaron valores entre 0.7 y 0.9 para dicha relación, en tanto que Guerrero et al. (2011) indicaron valores de 1.2. En las colectas evaluadas se detectaron frutos de forma oblato (0.7), es decir más anchos que largos, circular (1.0) y elíptico (1.5).

El componente del color de mayor variación fue el matiz $(\mathrm{CV}=46.4 \%)$, lo que se atribuye a que los frutos evaluados variaron de color rojo (15.4), al naranja (50-70), amarillo (80-90) y hasta con tendencia al color verde (105.4) (Cuadro 2). Ruenes-Morales et al. (2010) describieron los 
Cuadro 1. Materiales evaluados de ciruela mexicana (Spondias purpurea) y localización geográfica del lugar de colecta.

\begin{tabular}{|c|c|c|c|c|c|c|}
\hline Codificación & Estado & Municipio & \multicolumn{2}{|c|}{ Coordenadas (LN, LO) } & Altitud (m) & Nombre común ${ }^{\dagger}$ \\
\hline $\mathrm{CH} 1-\mathrm{CH} 8$ & Chiapas & Chiapa de corzo & $16^{\circ} 42^{\prime} 25^{\prime \prime}$ & $93^{\circ} 00^{\prime} 50^{\prime \prime}$ & 420 & $\begin{array}{l}\text { Chapilla, Jocote grande, Jocote de } \\
\text { ostuta, Jocote de azúcar, Jocote } \\
\text { de agua, Roja dulce }\end{array}$ \\
\hline GRO1-GRO6 & Guerrero & Taxco & $18^{\circ} 36^{\prime} 25.9^{\prime \prime}$ & $99^{\circ} 29^{\prime} 15.3^{\prime \prime}$ & 1154 & $\begin{array}{l}\text { Anaranjada agridulce, ácida, } \\
\text { Amarilla dulce, Roja dulce }\end{array}$ \\
\hline GRO7- GRO13 & Guerrero & Iguala & $18^{\circ} 20^{\prime} 41^{\prime \prime}$ & $99^{\circ} 32^{\prime} 34^{\prime \prime}$ & 732 & $\begin{array}{l}\text { Roja dulce uva, dulce (uva pe- } \\
\text { queña), amarilla dulce, mediana }\end{array}$ \\
\hline MOR1 & Morelos & Coatlán del Río & $18^{\circ} 45^{\prime} 35.5^{\prime \prime}$ & $99^{\circ} 27^{\prime} 28.8^{\prime \prime}$ & 1055 & Criolla roja ácida \\
\hline MOR2-MOR3 & Morelos & Tetecala & $18^{\circ} 43^{\prime} 50.7^{\prime \prime}$ & $99^{\circ} 24^{\prime} 8.4^{\prime \prime}$ & 1000 & Criolla roja dulce \\
\hline $\begin{array}{l}\text { MOR4-MOR7, } \\
\text { MOR20-MOR23 }\end{array}$ & Morelos & Jojutla & $18^{\circ} 34^{\prime} 23.9^{\prime \prime}$ & $99^{\circ} 15^{\prime} 51.4^{\prime \prime}$ & $935-961$ & $\begin{array}{l}\text { Criolla amarilla ácida, Criolla } \\
\text { roja dulce, Silvestre roja ácida, } \\
\text { Silvestre roja dulce, Roja dulce, } \\
\text { Amarilla dulce, Roja agridulce }\end{array}$ \\
\hline $\begin{array}{l}\text { MOR8-MOR13, } \\
\text { MOR19 }\end{array}$ & Morelos & Tlatizapán & $18^{\circ} 45^{\prime} 52.3^{\prime \prime}$ & $99^{\circ} 07^{\prime} 04.6^{\prime \prime}$ & 964 & $\begin{array}{l}\text { Cuauteca (roja dulce), Roja, Con- } \\
\text { servera dulce, Cuauteca (amarilla } \\
\text { dulce) }\end{array}$ \\
\hline $\begin{array}{l}\text { MOR14, } \\
\text { MOR17- MOR18 }\end{array}$ & Morelos & Puente de Ixtla & $18^{\circ} 37^{\prime} 29.2^{\prime \prime}$ & $99^{\circ} 19^{\prime} 23.9^{\prime \prime}$ & $901-1037$ & $\begin{array}{l}\text { Cuauteca (amarilla dulce), Roja } \\
\text { ácida }\end{array}$ \\
\hline MOR15-MOR16 & Morelos & Axochiapan & $18^{\circ} 33^{\prime} 18.2^{\prime \prime}$ & $98^{\circ} 46^{\prime} 35.6^{\prime \prime}$ & 1006- 1090 & $\begin{array}{l}\text { Roja ácida, Naranja ácida, Roja } \\
\text { ácida }\end{array}$ \\
\hline MOR24-MOR35 & Morelos & Tlalquitenango & $18^{\circ} 31^{\prime} 15.6^{\prime \prime}$ & $99^{\circ} 07^{\prime} 07.0^{\prime \prime}$ & 846- 1096 & $\begin{array}{l}\text { Roja dulce, Bolsona ácida, Roja } \\
\text { agridulce, Roja silvestre, Roja } \\
\text { ácida, Conservera }\end{array}$ \\
\hline MOR36-MOR 38 & Morelos & Mazatepec & $18^{\circ} 40^{\prime} 13.5^{\prime \prime}$ & $99^{\circ} 22^{\prime} 40.6^{\prime \prime}$ & $956-963$ & Roja dulce, \\
\hline MOR39- MOR43 & Morelos & Miacatlán & $18^{\circ} 43^{\prime} 19.9^{\prime \prime}$ & $99^{\circ} 18^{\prime} 51.3^{\prime \prime}$ & $978-1028$ & $\begin{array}{l}\text { Roja ácida silvestre, Roja agridul- } \\
\text { ce, Roja dulce }\end{array}$ \\
\hline MOR44- MOR46 & Morelos & Tetecala & $18^{\circ} 43^{\prime} 50.8^{\prime \prime}$ & $99^{\circ} 23^{\prime} 58.4^{\prime \prime}$ & $978-984$ & Amarilla dulce, Roja dulce \\
\hline
\end{tabular}

${ }^{\dagger}$ Nombre común indicado por personas cercanas al lugar de colecta.

Cuadro 2. Descriptores de características cuantitativas y parámetros estadísticos de la muestra de frutos de ciruela mexicana (Spondias purpurea).

\begin{tabular}{|c|c|c|c|c|c|c|}
\hline Variable & Media & $S^{2}$ & $\mathrm{r}$ & Mínimo & Máximo & $\mathrm{CV}(\%)$ \\
\hline Masa (g) & 10.6 & 40.6 & 24.5 & 4.0 (GRO6) & $43.2(\mathrm{CH} 3)$ & 60.1 \\
\hline Diámetro polar (mm) & 28.1 & 33.8 & 21.9 & 20 (GRO12) & $46.4(\mathrm{CH} 6)$ & 20.6 \\
\hline Diámetro longitudinal (mm) & 24.8 & 30.1 & 18.2 & $17.9(\mathrm{MOR} 43)$ & $42.8(\mathrm{CH} 4)$ & 22.0 \\
\hline $\begin{array}{l}\text { Forma (Diámetro polar/ } \\
\text { diámetro longitudinal) }\end{array}$ & 1.1 & 0.004 & 0.38 & $0.77(\mathrm{CH} 1)$ & 1.59 (MOR32) & 6.04 \\
\hline Luminosidad $\left(\mathrm{L}^{\star}\right)$ & 39.6 & 66.1 & 32.2 & $23.2(\mathrm{GRO} 4)$ & $62.6(\mathrm{CH} 4)$ & 20.5 \\
\hline Cromaticidad $\left(\mathrm{C}^{\star}\right)$ & 31.9 & 55.0 & 36.1 & $13.5(\mathrm{MOR} 23)$ & $60.3(\mathrm{CH} 4)$ & 23.3 \\
\hline $\operatorname{Matiz}\left(\mathrm{H}^{\star}\right)$ & 76.0 & 502.8 & 77.0 & 15.4 (MOR39) & $105.4($ GRO12) & 47.7 \\
\hline Sólidos solubles totales ( ${ }^{\circ}$ Brix) & 10.2 & 9.1 & 10.8 & $3.2(\mathrm{MOR} 38)$ & 17.3 (MOR12) & 29.5 \\
\hline Acidez titulable (\%) & 0.85 & 0.3 & 1.6 & 0.2 (MOR33) & $2.0(\mathrm{MOR} 40)$ & 62.2 \\
\hline Índice de sabor (SST/AT) & 17.6 & 135.6 & 37.9 & 3.0 (MOR40) & $63.2(\mathrm{MOR} 21)$ & 66.0 \\
\hline
\end{tabular}


colores rojo, amarillo, morado o guinda; en tanto que Ramírez et al. (2008), mencionaron colores como rojo/ tinta o amarillo-naranja. El color de la epidermis de la ciruela mexicana se atribuye a fenoles y carotenoides, que pueden ser de interés debido a la evidencia de los efectos benéficos de algunos fenoles en la salud humana. Al respecto, Beserra et al. (2011) reportaron la moderada concentración de fenoles totales, antocianinas y actividad antioxidante en esta especie.

Los valores máximos de SST fueron de $17.3^{\circ} \mathrm{Brix}$, que son superiores a lo reportado para frutos del Centro-Occidente de México con $15.6^{\circ B}$ Brix (Ramírez et al., 2008), pero inferiores a frutos de Veracruz y Yucatán cuyos valores oscilaron entre 18 y $21.1{ }^{\circ}$ Brix, respectivamente (Nava-Kuri y Uscanga, 1979; Ruenes-Morales et al., 2010). Según, Koziol y Macía (1998), el aporte de energía de estos frutos es debido a la alta concentración de SST, de los cuales $65 \%$ corresponde a glucosa, fructosa y sacarosa.

La acidez titulable (AT) fluctuó entre 0.2 y 2.0 \% (Cuadro 2) mientras que Cunha et al. (2001) reportaron valores entre 0.6 y $0.9 \%$ de AT. El índice de sabor varió entre 3.0 y 63.2 (Cuadro 2); en esta variable Pérez et al. (2004) reportaron valores de 23.4 en frutos provenientes de Oaxaca. Según, Ladaniya (2008), la proporción SST/AT indica lo dulce o amargo de un fruto; así un incremento en el valor de la proporción corresponde a una disminución de la acidez.

El análisis de conglomerados, con un índice taxonómico medio de 1.2, se formaron siete grupos (Figura 1). En el Grupo I estuvieron 15 materiales, tres de Chiapas, dos de Guerrero y nueve de Morelos; en este grupo se ubicaron frutos de color naranja y amarillo con mayor pureza y brillo, de masa mediana, redondos y moderada proporción SST/AT (Cuadro 3). En el Grupo II se ubicaron dos materiales de Chiapas denominados 'Jocote de Ostuta' y 'Jocote de azúcar', con una forma más elíptica por tener diámetro polar mayor que el longitudinal; sus características de SST y AT fueron muy similares a las del grupo anterior (Cuadro 3). En el Grupo III estuvieron los 20 materiales de menor masa, SST y mayor acidez, de color rojo puro y brillante, 90 $\%$ de estos materiales provienen de Morelos (Cuadro 3).
En el Grupo IV estuvieron ocho materiales de color rojo opaco y brillante, con bajos SST y AT, masa intermedia, y valores mayores de diámetro polar que ecuatorial (Cuadro 3). El Grupo V se conformó de frutos de color rojo puro y brillante, con la mayor proporción SST/AT y de masa baja; principalmente procedieron de Guerrero y Morelos. En el Grupo VI se ubicaron dos materiales de color amarillo opaco, de masa baja y sabor dulce provenientes de Guerrero. Finalmente, en el Grupo VII se ubicaron los frutos de 'Jocote grande', proveniente de Chiapas, que son los de tamaño mayor, de forma redonda con tonalidades entre naranja y amarilla puro, brillante y sabor dulce (Cuadro 3).

Los frutos de ciruela mexicana pueden emplearse para consumo en fresco y para la elaboración de nieves, salsas, postres, mermeladas, deshidratados, confituras, licores y aguas (Perez et al., 2008). En la amplia variabilidad encontrada en el presente trabajo, destacan materiales con potencial para su comercialización en fresco, como son los ubicados en los Grupos I, IV, V y VII, que poseen altos índices de sabor y son de color amarillo y rojo. Según, Ruenes-Morales et al. (2010), $90 \%$ de las personas prefieren la ciruela mexicana dulce y con gran proporción de pulpa. Los materiales de los Grupos III y VI, por sus valores altos de acidez pueden usarse para hacer conservas y salsas. En ambos casos es necesario efectuar más estudios sobre el contenido y caracterización de antioxidantes, para que su consumo aporte más beneficios. Finalmente, es necesario conocer, conservar y proteger esta especie tropical con potencial frutícola como recurso fitogenético del país.

\section{CONCLUSIONES}

Se determinó variabilidad alta en las colectas aquí evaluadas de ciruela mexicana, que se diferencian en siete grupos. Las variables que ayudaron a su separación fueron masa, dimensiones y color (ángulo matiz) de la epidermis.

\section{AGRADECIMIENTOS}

Se agradece el apoyo de SEP-PROMEP a la Red "Ciencia y Tecnología Pre y Postcosecha” para la publicación del presente trabajo. 


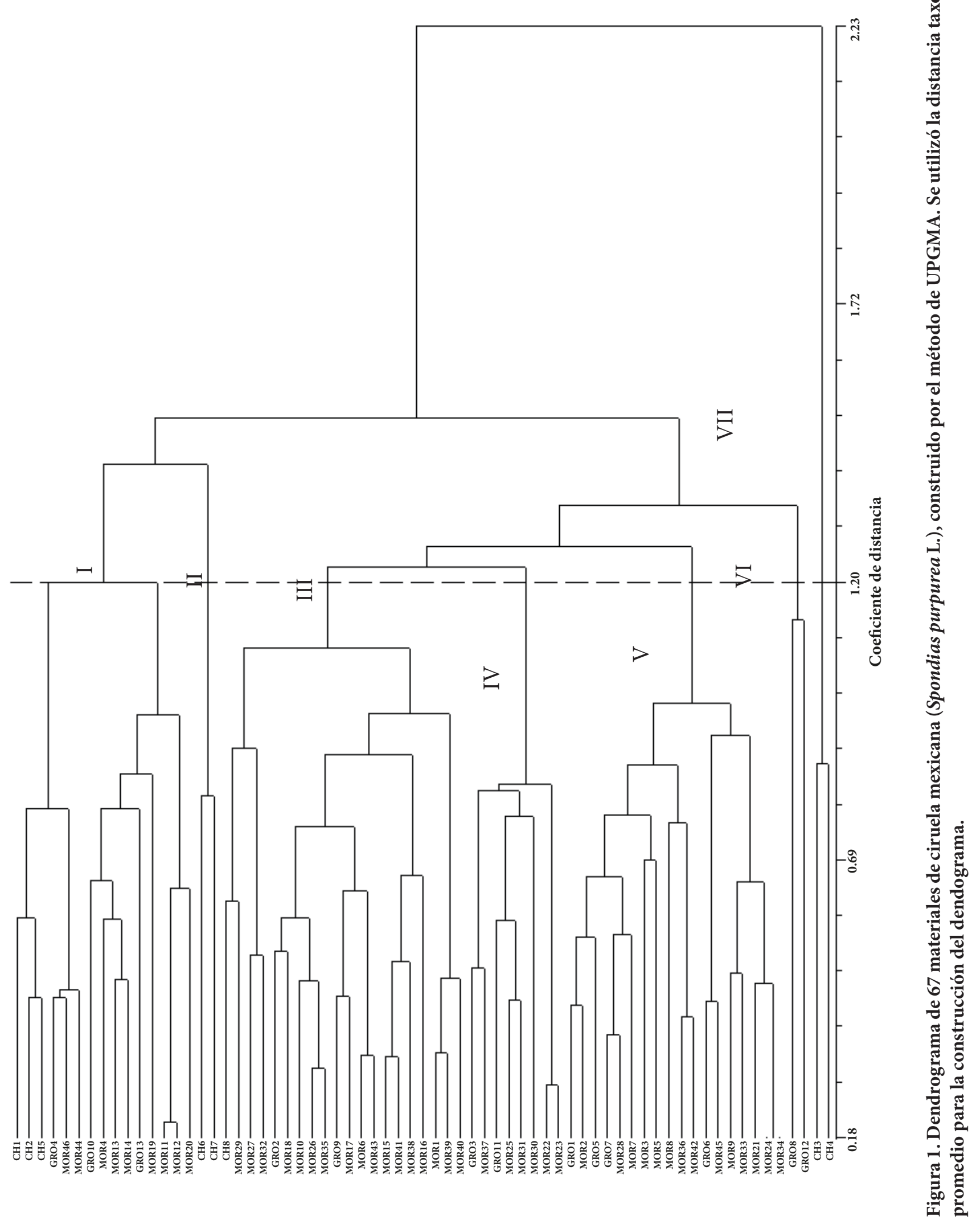


Cuadro 3. Promedios de las variables evaluadas en los siete grupos formados por el análisis de conglomerados de 67 materiales de ciruela mexicana (Spondias purpurea).

\begin{tabular}{ccccccccccc}
\hline Grupo & Masa (g) & $\begin{array}{c}\text { Diámetro } \\
\text { polar (mm) }\end{array}$ & $\begin{array}{c}\text { Diámetro } \\
\text { longitudinal }(\mathrm{mm})\end{array}$ & $\begin{array}{c}\text { Índice } \\
\text { de forma }\end{array}$ & $\mathrm{L}^{*}$ & $\mathrm{C}^{*}$ & $\mathrm{H}^{*}$ & $\begin{array}{c}\text { Sólidos solubles } \\
\text { totales }\left({ }^{\circ} \text { Brix) }\right.\end{array}$ & $\begin{array}{c}\text { Acidez } \\
\text { titulable }(\%)\end{array}$ & $\begin{array}{c}\text { Índice de } \\
\text { sabor }\end{array}$ \\
\hline I & 15.0 & 30.7 & 28.4 & 1.1 & 50.8 & 40.3 & 74.4 & 11.7 & 0.6 & 19.1 \\
II & 20.7 & 40.9 & 29.7 & 1.4 & 46.2 & 30.9 & 87.2 & 11.4 & 0.6 & 17.9 \\
III & 9.5 & 27.9 & 23.6 & 1.2 & 64.4 & 30.3 & 37.5 & 9.7 & 1.0 & 11.0 \\
IV & 17.8 & 33.7 & 29.4 & 1.1 & 36.5 & 27.7 & 32.2 & 9.7 & 0.6 & 18.3 \\
V & 9.7 & 28.2 & 23.8 & 1.2 & 33.6 & 29.2 & 29.7 & 11.9 & 0.4 & 31.0 \\
VI & 11.2 & 29.2 & 25.2 & 1.1 & 40.7 & 19.2 & 88.8 & 10.9 & 0.7 & 14.3 \\
VII & 33.6 & 40.0 & 38.5 & 1.0 & 58.3 & 50.9 & 75.8 & 15.0 & 0.8 & 18.5 \\
\hline
\end{tabular}

$\mathrm{L}^{*}=$ luminosidad (0: blanco, 100: negro); $\mathrm{H}^{*}=$ ángulo matiz (0: rojo; 90: amarillo); $\mathrm{C}^{\star}=$ cromaticidad (del gris).

\section{BIBLIOGRAFÍA}

Avitia G E, Castillo G A M, Pimienta B E (2000) Ciruela mexicana y otras especies del genero Spondias L. Universidad Autónoma Chapingo. Chapingo, Estado de México, México. 75 p.

Bautista-Baños S, J C Díaz-Pérez, L L Barrera-Necha, L Bravo-Luna (2003) Estudio postcosecha de la ciruela mexicana (Spondias purpurea L.) durante almacenamiento. Rev. Iber. Tecnología Postcosecha 5:82-85.

Bautista B S, M Hernández L, D Guillén S, I Alia T (2006) Influencia del recubrimiento con quitosano y la temperatura en la calidad postcosecha y niveles de infección en la ciruela mexicana. Rev. Iber. Tecnología Postcosecha 7:114-121.

Beserra A M M, P E Machado de S, A M Campos A, G Matias do P, C E de Carvalho M, G Arraes M, T L Gomes de L (2011) Bioactive compounds and the antioxidant activity of fresh exotic fruits from northeastern Brazil. Food Res. Internat. 44:2155-2159.

Cunha F H A, R E. Alves, C F Herbster M, A C de Oliveira, N C Costa A (2001) Calidad de frutas nativas de latinoamérica para industria: ciruela mexicana (Spondias purpurea L.) Proc. Interamer. Soc. Trop. Hort. 43:68-71.

Gaona-García A, I Alia-Tejacal, V López-Martínez, M Andrade-Rodríguez, M T Colinas-León, O Villegas-Torres (2008) Caracterización de frutos de zapote mamey (Pouteria sapota) en el Suroeste del estado de Morelos. Rev. Chapingo S. Hort. 14:41-47.

Guerrero, R, M Manzanilla, C Hernández, J. Chacín, C Clamens (2011) Caracterización fisicoquímica de frutos ciruelo de huesito (Spondias purpurea L.) en el municipio de Mara Rev. Fac. Agron. (LUZ) 27 (Supl. 1):670-676.

Koziol J M, J Macía M (1998) Chemical composition, nutritional evaluation, and economic prospects of Spondias purpurea (Anacardiaceae). Econ. Bot. 52:373-380.

Ladaniya S M (2008) Citrus Fruits. Biology, Technology and Evaluation. Academic Press. San Diego, California, USA. 558 p.
Macia M J, A Badfor S (2000) Economic Botany of Spondias purpurea (Anacardiaceae) in Ecuador. Econ. Bot. 54:449-458.

McGuire R G (1992) Reporting of objective color measurements. HortScience 27:1254-1255.

Nava K G, M Uscanga B (1979) Estudio físico y químico de doce tipos de ciruela (Spondias sp.) en el estado de Veracruz. Proc Tropical Recion ASHS 23: 132-136.

Nava-Kuri G, M. Uscanga B (1979) Estudio físico y químico de doce tipos de ciruela (Spondias sp.) en el estado de Veracruz. Proc. Trop. Region ASHS 23:132-136.

Osuna G J A, M H Pérez B, V Vázquez V, R Gómez J (2011) Aplicación de 1-metilciclopropeno (1-MCP) y su efecto en ciruela mexicana. Rev. Fitotec. Mex. 34:197-204.

Pérez L A, C Saucedo V, M L Arevalo G, A Muratalla L. (2004)Efecto del grado de madurez en la calidad y vida poscosecha de ciruela mexicana (Spondias purpurea L.). Rev. Fitotec. Mex. 27:133139.

Pérez-Arias A G A, I Alia-Tejacal, M Andrade-Rodríguez, V LópezMartínez, A Pérez-López L, R Ariza-Flores, M A Otero-Sánchez, J M Villarreal-Fuentes (2008) Características físicas y químicas de ciruelas mexicana (Spondias purpurea) en Guerrero. Inv. Agropec. 5:141-149.

Ramírez H B C, E Pimienta B, J Z Castellanos R, a Muñoz U, G Palomino H, E Pimienta B (2008) Sistemas de producción de Spondias purpurea (Anacardiaceae) en el centro-occidente de México. Rev. Biol Trop. 56:675-687.

Ruenes-Morales M del R, A Casas, J J Jiménez-Osornio, J Caballero (2010) Etnobotánica de Spondias purpurea L. (Anacardiaceae) en la península de Yucatán. Interciencia 35:247-254.

SAS Institute (1998) SAS User's guide: Statics. Version 6.12. SAS Institute, Cary, N. C. 1848 p.

Vargas Simón G, R Hernández-Cupil, E Moguel-Ordoñez (2011) Caracterización morfológica de ciruela (Spondias purpurea L.) en tres municipios del estado de Tabasco, México. Bioagro 23:141149. 